\title{
Cannabisabhängigkeit: eine ernstzunehmende Erkrankung
}

$\mathrm{D}$ ie öffentliche Diskussion um suchtstoffbezogene Erkrankungen betraf in den letzten Jahrzehnten vor allem die Alkoholabhängigkeit und die Opiatabhängigkeit. Entsprechend sind Behandlungseinrichtungen auch vorwiegend auf die von solchen Suchtmitteln Abhängigen ausgerichtet. Cannabisabhängigkeit spielte hier lange keine Rolle. Zudem war die öffentliche Diskussion um den Konsum von Cannabis geprägt durch die so genannte Legalisierungsdebatte: hierbei wurde von Befürwortern einer Verkehrsfähigkeit von Cannabis behauptet, dass ein gelegentlicher Freizeitkonsum gesundheitlich harmlos sei und die rechtlichen Einschränkungen zur Verfügbarkeit von Cannabis eine Kriminalisierung jugendlicher Konsumenten bedeute. In der Fachdiskussion wurde dem gegenüber die Bedeutung des Cannabiskonsums als eines eigenständigen Risikofaktors für die Entwicklung einer schizophrenen Psychose intensiv beforscht und kontrovers diskutiert.

Nach den epidemiologischen Entwicklungen der letzten zehn bis zwanzig Jahren müssen wir die Cannabisdiskussion verändern. Wir müssen zur Kenntnis nehmen, dass eine Minderheit der Cannabiskonsumenten nicht bloß einen Freizeitkonsum betreibt, sondern täglich konsumiert. Ein Teil dieser Konsumenten ist bei Anwendung der ICD-10-Kriterien für das Abhängigkeitssyndrom cannabisabhängig, also nach Anwendung der gleichen Kriterien wie bei der Diagnose der Alkohol- und Opiatabhängigkeit. Die aktuelle Zahl der Cannabisabhängigen in Deutschland wird auf über 200000 Personen geschätzt. Im Kontext solcher Zahlen erscheint in der politischen Diskussion eine argumentativ unveränderte Befürwortung der Legalisierung von Cannabis leichtfertig. Auch die Engführung der Fachdiskussion auf den Zusammenhang von Cannabis und Psychose wird dem aktuellen Problem nicht gerecht.

Vor dem skizzierten Hintergrund werden in diesem Heft von psychoneuro schwerpunktmäßig Darstellungen zur Cannabisabhängigkeit zu lesen sein. Professor Dr. U. Schneider (Lübbecke) gibt einen Überblick über die biologische Forschung zu Endocannabinoiden. Dies ist der Hintergrund für das Verständnis der Wirkungen des Suchtmittels Cannabis. Herr PD Dr. U. Preuss (Greifswald) befasst sich mit dem Cannabisentzugssyndrom, einem klinischen Beschwerdebild, dessen Existenz noch vor wenigen Jahren auch von Fachkollegen grundsätzlich bestritten wurde. Herr Dr. N. Quecke (Essen) sowie Herr PD Dr. U. Bonnet (Essen) befassen sich mit der Therapie der Cannabisabhängigkeit im Jugend- bzw. im Erwachsenenalter. Neben anderem ist aus diesen Übersichten ersichtlich, dass die Behandlung der Cannabisabhängigkeit weitgehend auf Evidenzen aus den USA und Australien angewiesen ist. Angesichts der soziokulturellen Unterschiede zwischen den genannten Ländern und Deutschland ist ein solcher Transfer von Behandlungserfahrungen nur begrenzt zulässig - trotz aller Bedeutsamkeit solcher Untersuchungen. Untersuchungen zur Behandlung der Cannabisabhängigkeit in Deutschland sind in wissenschaftlicher wie in klinisch-therapeutischer Perspektive von hoher Bedeutung.

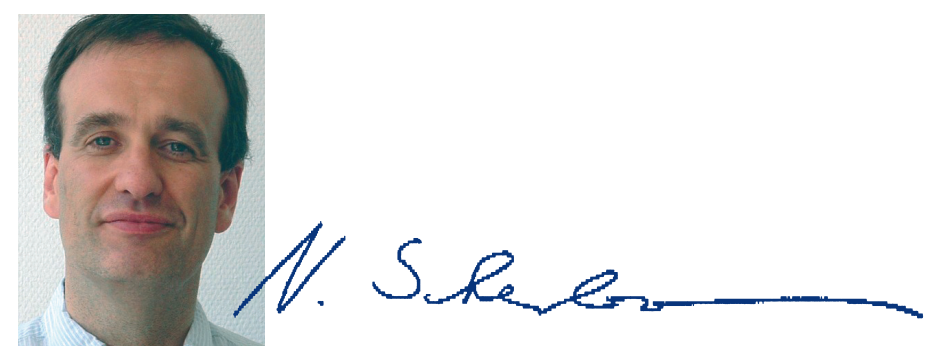

Prof. Dr. med. Norbert Scherbaum, Essen 